\title{
Sistema de gestión de información para el Consultorio Jurídico y Centro de Conciliación de la Universidad de Ibagué
}

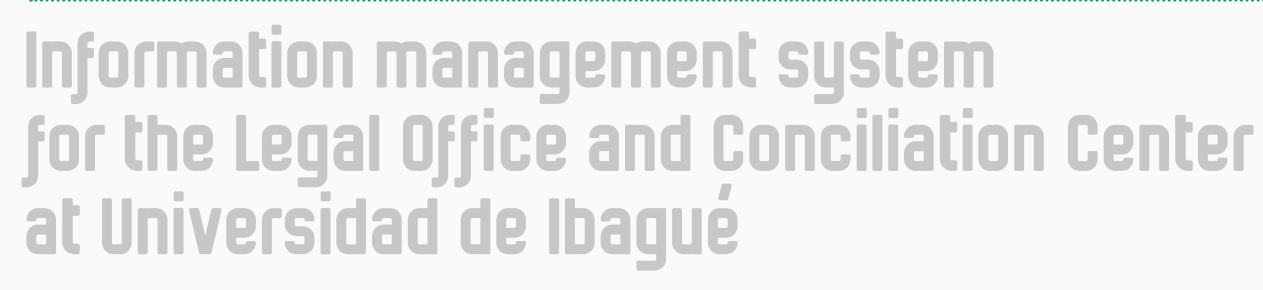

\author{
Andrés A. Garcia León ${ }^{1}$ \\ Dayanne A. González Villanueva² \\ Juliana Martínez Higuita ${ }^{3}$ \\ William E. Torres Tapia" \\ Sandra Bibiana Vargas Castro ${ }^{5}$
}

(2).

Recepción: 03/10/2020
(2)

Aprobación: 15/10/2020

Publicación: 18/12/2020

Para citar este artículo:

García León, A. A., González Villanueva, D. A., Martínez Higuita, J., Torres Tapia, W. F., \& Vargas Castro, S. B. (2020). Sistema de gestión de información para el Consultorio Jurídico y Centro de Conciliación de la Universidad de Ibagué. Indagare, (8),178-187. https://doi.org/10.35707/indagare/815

\section{(c) $(1) \Theta(\Theta$}

\footnotetext{
${ }^{1}$ Grupo de investigación GINNOvA, Universidad de Ibagué, Colombia. ORCID: 0000-0003-1757-6885. Correo electrónico: andres.garcia@unibague.edu.co

${ }^{2}$ Grupo de investigación GINNOvA, Universidad de Ibagué, Colombia. ORCID: 0000-0001-6708-9212. Correo electrónico: dayanne.gonzalezv@gmail.com

${ }^{3}$ Grupo de investigación GINNOva, Universidad de Ibagué, Colombia. ORCID: 0000-0002-1969-9929. Correo electrónico: juliana.martinez99@hotmail.com

${ }^{4}$ Grupo de investigación GINNOva, Universidad de Ibagué, Colombia. ORCID: 0000-0001-5874-3091. Correo electrónico: william.torres@unibague.edu.co

${ }^{5}$ Grupo de investigación Zoon Politikon, Universidad de Ibagué, Colombia. ORCID: 0000-0002-7308-8977. Correo electrónico: sandra.vargas@unibague.edu.co
} 


\title{
Resumen
}

El Consultorio Jurídico y Centro de Conciliación de la Universidad de Ibagué ha brindado su servicio por 21 años y ha cumplido con el proyecto social y educativo de la institución a través de la asistencia legal gratuita a personas de estratos 1 y 2 . Desde el inicio de operaciones en el año 1999, la información correspondiente a la trazabilidad de los casos se gestiona por medio de registros físicos, que dificultan la elaboración de informes e indicadores. De acuerdo con lo anterior, el presente artículo expone el desarrollo de un sistema de gestión de información que facilite el registro y la elaboración de informes e indicadores en tiempo real. Para ello, se analizaron los procedimientos de todas las dependencias y la gestión de datos actual, con el fin de obtener los requerimientos necesarios para el eficiente funcionamiento del Consultorio Jurídico y Centro de Conciliación. Una vez se conoció la trazabilidad de los datos, se procedió con la creación de un software que facilitara la comunicación, optimización del funcionamiento y estandarización de los procesos.

\begin{abstract}
The Legal Office and Conciliation Center at Universidad de Ibagué has provided its service for 21 years and has complied with the institution's social and educational project through free legal assistance to people from socio-economic status 1 and 2. Since the beginning of operations in 1999, the information corresponding to the traceability of the cases is managed through physical records, which make it difficult to prepare reports and indicators. In accordance with the above, this article presents the development of an information management system that facilitates recording and preparation of reports and indicators in real time. To do this, the procedures of all agencies and current data management were analyzed, in order to obtain the necessary requirements for the efficient operation of the Legal Office and Conciliation Center. Once the traceability of the data was known, the creation of a software was undertaken, which facilitated communication, optimization of the operation and standardization of the processes.
\end{abstract}

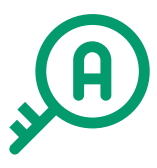

Palabras claves: Sistema de información, servicio, modelo, trazabilidad, eficiencia y mejora continua.

Key words: Information system, service, model, traceability, efficiency, continuous improvement. 


\section{Introducción}

El Consultorio Jurídico y Centro de Conciliación de la Universidad de Ibagué está comprometido en asesorar, direccionar y acompañar procesos legales, además familiariza a los estudiantes de los cuatro últimos semestres del Programa de Derecho en la práctica del ejercicio profesional. Sin embargo, a pesar de que ha ofrecido a la comunidad un buen servicio, no cuenta con un modelo de gestión en el que se especifique cómo deben realizarse las tareas de manera eficiente, y en el que se integren sistemas que admitan conocer la evolución e información de los casos en el momento requerido.

Lo anterior ha generado que la prestación del servicio a sus usuarios no se brinde de forma eficiente por el complejo trabajo de consulta en los libros, por ejemplo, para solicitar información sobre el estado y la asignación de los procesos. Además, la falta de información ha impedido la elaboración de análisis estadísticos que expongan el aumento o la disminución de las cifras en diferentes casos y, de esta forma, aplicar medidas necesarias para el control y mejoramiento de la calidad de vida de los ciudadanos.

Todos los registros que se toman en el Consultorio Jurídico y Centro de Conciliación, desde la recepción de los consultantes, hasta el cierre de la carpeta del proceso, se hacen de forma manual, es decir, son consignados en libros o formularios que generan pérdida de tiempo en las actividades relacionadas con su consulta, desigualdad en el número de casos asignados a los estudiantes e ineficiencia para dar solución a los mismos. Por ejemplo, por asignar casos que ya están siendo asistidos, pero que, por la dificultad de hallar su información, se opta por reasignarlos a otros estudiantes. De esta manera se genera confusión, sobresfuerzos en su asistencia y demoras en la atención del consultorio; acciones que crean una baja satisfacción entre los usuarios.

Hoy en día existen múltiples mecanismos tecnológicos que apoyan el manejo y la trazabilidad de los datos, como los sistemas de gestión de información. Estos elementos interactúan entre sí por medio de un recurso computacional para facilitar el manejo e interpretación de la información (INCAP, s.f.). El objetivo latente es ayudar al desempeño de las actividades que desarrolla la empresa, suministrando la información adecuada, con la calidad requerida, a la persona o departamento que lo solicita, en el momento y lugar especificados con el formato más útil para el receptor (Universidad Politécnica de Cataluña, 2004).

Con la implementación de un sistema de gestión de información, las organizaciones ahorran esfuerzo y costos al tramitar digitalmente los documentos, de esta manera, se eliminan las tareas repetitivas y el uso del papel. Así aumenta el grado de satisfacción, puesto que las actividades diarias son más fáciles de ejecutar, se agiliza 
el servicio y se promueve que los clientes estén más satisfechos. Por último, propicia el conocimiento detallado de la información en tiempo real, la mantiene actualizada y facilita tomar decisiones acertadas en cada proceso (Siigo, 2018).

Dentro de la búsqueda de sistemas de gestión de información que son usados en oficinas de abogados, bufetes y consultorios jurídicos, se encontraron diversos softwares que son implementados para solucionar requerimientos específicos. Algunos ejemplos relevantes a nivel nacional son Lege (García, Flórez \& Olier, 2015), Redelex (Redelex, s.f.) y Legisoffice (Legisoffice, 2014).

Finalmente, al percibir la importancia de implementar un sistema de gestión de la información en diversas organizaciones y los problemas que presenta el manejo y la trazabilidad de los datos en el consultorio jurídico de la Universidad, el objetivo de esta investigación es desarrollar un sistema de gestión de información que cumpla con todos los requerimientos necesarios para manejar los datos y su trazabilidad de la manera más idónea.

\section{Materiales y métodos}

Para el desarrollo de este proyecto, inicialmente se caracterizaron los procesos del Consultorio Jurídico y Centro de Conciliación y se ejecutó una revisión documental en su reglamento interno para identificar su filosofía corporativa, estructura organizacional y funciones de todo el personal encargado de su funcionamiento. Además, se realizó una serie de visitas para la observación directa de los procedimientos que se ejecutan a diario, utilizando herramientas tales como organigramas y diagramas de flujo para simplificar la información.

Después de caracterizar los procesos y procedimientos, se obtuvieron los requerimientos necesarios para el buen funcionamiento del consultorio. Para este paso, se realizaron una serie de visitas en las que se revisaron las variables depositadas en los libros de cada una de las dependencias. Después se inició con el desarrollo de la herramienta computacional que gestionaría los datos y, para ello, se emplearon las siguientes actividades:

- Diseño del sistema: toda la información recogida se intentó plasmar mediante la descripción de la estructura relacional global del sistema y la especificación de aquello que debió hacer cada una de sus partes, así como la manera en que se combinaron unas con otras, mediante el uso del lenguaje unificado de modelado UML (Unified Modeling Language por sus siglas en inglés).

- Implementación: es la fase en la que se implementó el código fuente. Se usaron prototipos, pruebas y ensayos para corregir errores. Al ser un aplicativo 
INDAGA3E e-ISSN: 2357-5042 • Número 8 (2020) • Universidad de Ibagué • doi: https://doi.org/10.35707/indagare/815

para escritorio, se utilizó el entorno de desarrollo NetBeans y el lenguaje de programación Java. Se implementó una base relacional y administrada por el sistema de gestión de base de datos MySQL, que será alojada en un servidor local.

- Validación: se compiló el software y se comprobó su correcto funcionamiento. Se buscaron sistemáticamente y se corrigieron todos los errores antes de que fuera entregado al usuario final.

\section{Resultados preliminares}

Los resultados obtenidos para esta investigación están relacionados con la caracterización de la prestación del servicio, la presentación de los requerimientos y la pantalla principal del software CONSULSOFT.

\subsection{Caracterización de la prestación del servicio}

Cuando un usuario ingresa al consultorio es atendido por el coordinador de turno que corresponde con el estudiante que recibe la información. El coordinador verifica si el asunto que se presenta es competencia de la dependencia y si el solicitante debe atenderse debido a su condición económica. En caso afirmativo, el usuario se dirige a los cubículos y entrega la información requerida para el diligenciamiento de un formulario, documento que al finalizar el día es entregado por el coordinador de turno al área respectiva.

De acuerdo con lo anterior, se asigna el estudiante que por reparto debe asumir el seguimiento y resolución del caso. En consecuencia, el estudiante cita a las partes por medio de comunicación dirigida a la dirección registrada en la petición; en ella señala el lugar, la fecha y la hora en las que se llevará a cabo la reunión respectiva. En todo caso deberá ser convocada, a más tardar, dentro de los ocho días hábiles siguientes, salvo que por razones especiales de distancia o ausencia de una de las partes no lo permitan. En caso contrario, el usuario debe dirigirse a los cubículos donde otro coordinador de turno le explica las razones por las cuales no se atenderá su caso. Sin embargo, se le brinda una asesoría y si fuere necesario, tiene la posibilidad de acudir al monitor, asesor de área o director del consultorio, para replantear la negativa.

El diagrama de flujo que se presenta en la Figura 1 se diseñó para generar mayor claridad frente al proceso mencionado. Allí se describe la secuencia e interacción entre los diferentes eslabones que se encuentran dispuestos en el consultorio para brindar el servicio en la actualidad. 
Figura 1. Diagrama de flujo del Consultorio Jurídico y Centro de Conciliación de la Universidad de Ibagué

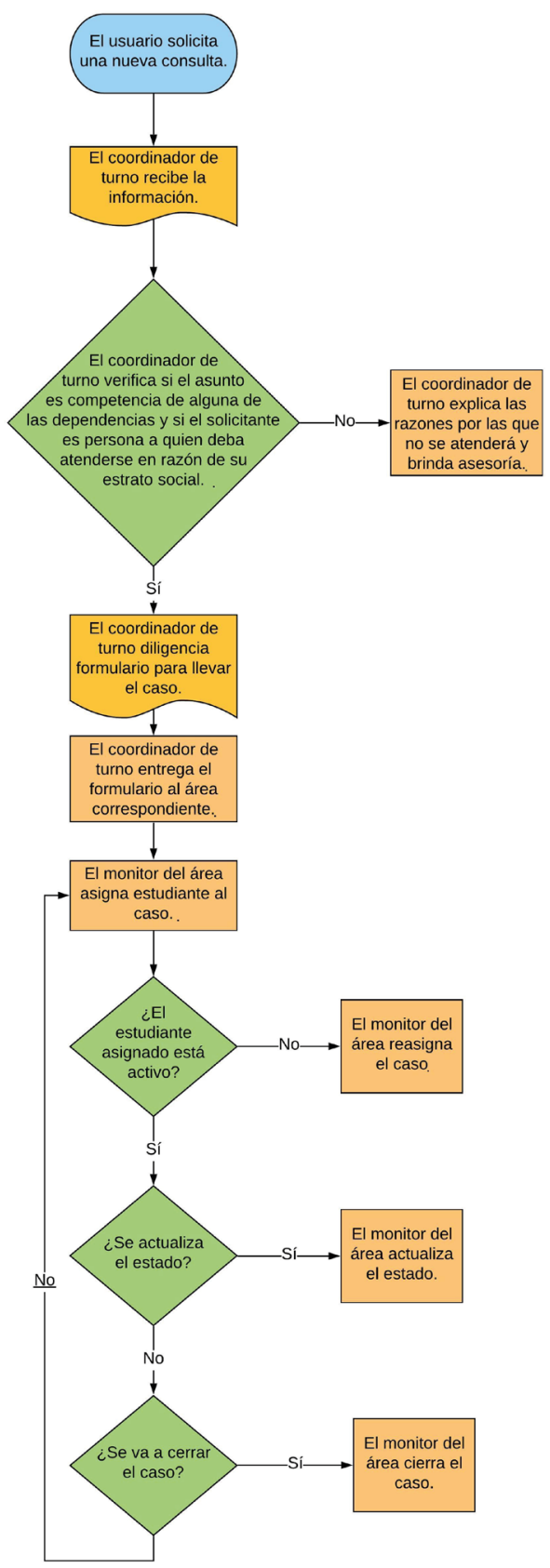

Fuente: elaboración propia 


\subsection{Requerimientos del sistema de información}

Con base en la caracterización de los procesos, al analizar los resultados se evidencia que en ningún momento se menciona la existencia de un sistema de información digital que agilice su desarrollo y la obtención de información en el consultorio. Por esta razón y al ser de gran importancia para el mejoramiento de la eficiencia en el servicio, se propone el uso de un sistema de información digital que reconozca la trazabilidad y el control de los procedimientos. De acuerdo con las características presentadas en el marco referencial de las herramientas utilizadas para el manejo de la información en otros consultorios, se tomó la decisión de diseñar y crear un software online que estuviera enlazado a una base de datos relacional.

Para el diseño de los formatos por utilizar en el software, se hizo necesario observar y comprender toda la información solicitada en la documentación manejada por el consultorio. Lo anterior se llevó a cabo con el fin de garantizar la obtención de información completa y con sentido. Por esta razón, se identificaron un conjunto de requerimientos funcionales (ver Tabla 1) y no funcionales del sistema, como el tiempo de respuesta en cada consulta, portabilidad, respaldo de los datos, facilidad de uso, facilidad de mantenimiento, etc. También, por medio de entrevistas no estructuradas aplicadas al personal del consultorio, se analizaron sus necesidades para la trazabilidad de los casos manejados.

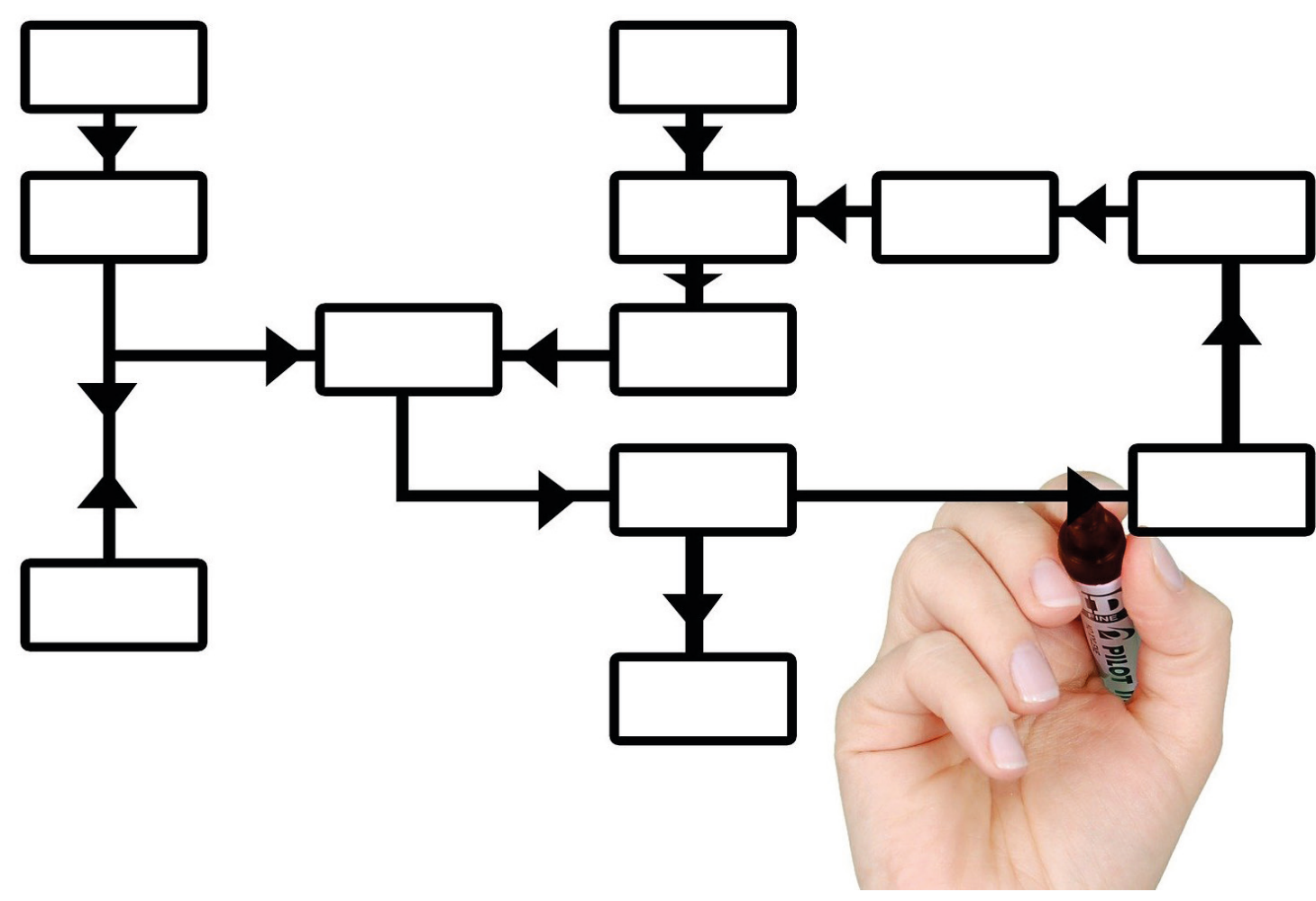


Tabla 1. Requerimientos funcionales

\begin{tabular}{|c|c|}
\hline Requerimiento & Descripción \\
\hline \multirow[t]{6}{*}{ Menú inicio } & Cambiar contraseña: permite cambiar la clave o \\
\hline & contraseña que fue ingresada durante el proceso de instalación. \\
\hline & Setup radicado: permite reiniciar el consecutivo según el semestre. \\
\hline & Editar galería: permite la edición de la galería fotográfica. \\
\hline & Cerrar sesión: permite cerrar la sesión. \\
\hline & Salir: permite salir de la sesión. \\
\hline \multirow[t]{3}{*}{ Menú estudiante } & Agregar estudiante: permite vincular a un estudiante al consultorio. \\
\hline & Eliminar estudiante: permite eliminar al estudiante cuando finaliza su práctica. \\
\hline & $\begin{array}{l}\text { Información sobre el estudiante: permite extraer información acerca de algún estudiante que } \\
\text { esté realizando las prácticas. }\end{array}$ \\
\hline \multirow[t]{8}{*}{ Menú coordinación } & $\begin{array}{l}\text { Agregar consulta: permite agregar una consulta a la base de datos, es decir, aquellos casos que } \\
\text { no podrán ser atendidos por el consultorio porque no es de su competencia o por el estrato } \\
\text { socioeconómico del usuario. }\end{array}$ \\
\hline & $\begin{array}{l}\text { Consultar libro de consultas: permite ver las consultas de un usuario por medio de su número } \\
\text { de cédula. }\end{array}$ \\
\hline & Ver libro de consultas: permite ver las consultas que han sido registradas en la base de datos. \\
\hline & Agregar radicado: permite agregar un radicado a la base de datos. \\
\hline & $\begin{array}{l}\text { Consultar libro de radicaciones: permite consultar cualquier radicado registrado en el libro de } \\
\text { radicaciones. }\end{array}$ \\
\hline & Ver libro de radicaciones: permite ver todos los radicados que han sido registrados. \\
\hline & Ficha bibliográfica: permite registrar la información bibliográfica de los usuarios. \\
\hline & $\begin{array}{l}\text { Consultar ficha bibliográfica: permite consultar la información de algún usuario que hubiere } \\
\text { sido previamente registrado. }\end{array}$ \\
\hline \multirow{11}{*}{$\begin{array}{l}\text { Menú } \\
\text { Centro de } \\
\text { Conciliación, } \\
\text { Clínica Jurídica, } \\
\text { Desplazados, } \\
\text { Derecho Penal, } \\
\text { Derecho Laboral, } \\
\text { Derecho Público y } \\
\text { Derecho Privado }\end{array}$} & Asignar caso: permite la asignación de los casos a los estudiantes. \\
\hline & Cambiar estado del caso: permite actualizar el estado en el proceso de los casos. \\
\hline & $\begin{array}{l}\text { Consultar libro de asignaciones: permite consultar quién es el estudiante encargado de llevar } \\
\text { determinado caso. }\end{array}$ \\
\hline & Ver libro de asignaciones: permite ver los casos asignados del área. \\
\hline & $\begin{array}{l}\text { Sustituir caso: permite cambiar a un estudiante por otro para que continúe con el proceso de } \\
\text { determinado caso. }\end{array}$ \\
\hline & Consultar libro de sustituciones: permite la consulta de las sustituciones realizadas a los casos a \\
\hline & través del proceso. \\
\hline & Ver libro de sustituciones: permite ver las sustituciones del área. \\
\hline & $\begin{array}{l}\text { Cerrar caso: permite cerrar los casos que por alguna razón ya han terminado su proceso en el } \\
\text { consultorio. }\end{array}$ \\
\hline & Consultar libro de cierre: permite consultar de manera directa si un caso ha sido cerrado. \\
\hline & Ver libro de cierre: permite ver los casos que han sido cerrados. \\
\hline
\end{tabular}

Fuente: elaboración propia 


\subsection{Pantalla principal del software}

Como resultado de las necesidades y requerimientos previamente analizados, se obtuvo el software CONSULSOFT, el sistema de información propuesto que unifica las consultas y procesos civiles, laborales, públicos, penales y de las unidades de clínica jurídica, conciliación y desplazados, codificado en el lenguaje Java e implementado en el ambiente de desarrollo Net-Beans 8.2.

Figura 2. Pantalla principal de CONSULSOFT

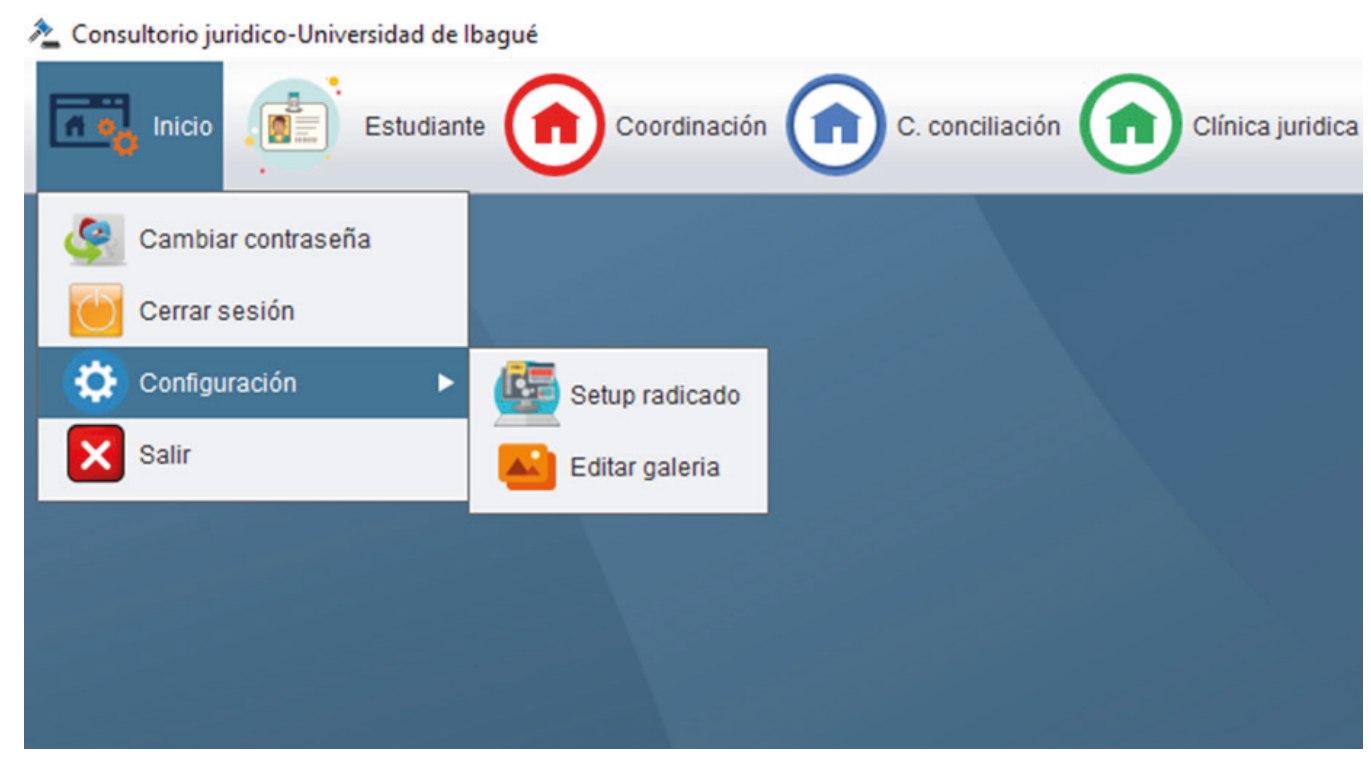

Fuente: elaboración propia

\section{Potencial uso}

CONSULSOFT es un sistema de gestión de información que se especializa en brindar un manejo y una trazabilidad adecuada a los datos generados por cualquier consultorio jurídico colombiano que desee modernizar sus sistemas de información. Si bien, en la actualidad es un software que funciona en la red local del Consultorio Jurídico y Centro de Conciliación de la Universidad de Ibagué, la base de datos se puede trasladar a un servidor de hosting dedicado. Esto hace que el software funcione desde otros equipos y se pueda instalar en consultorios con mayor número de usuarios. 


\section{Ficha técnica del proyecto}

Título del proyecto: Formulación y validación de heurísticas para optimizar el servicio al cliente en configuraciones flexibles en el departamento del Tolima.

PRIT: Industria de servicios de alto valor agregado y de talla internacional.

Código del proyecto: $17-465$-INT.

Palabras claves: Flexibilidad, Scheduling, servicio al cliente, competitividad, Job-shop, criterios regulares, pymes y Frente de Pareto.

Grupo de investigación: GINNOVA - Gestión e innovación empresarial

Investigador principal: Andrés Alberto García León.

Correo electrónico: andres.garcia@unibague.edu.co

\section{Referencias}

García, F., Flórez, N. D. C., \& Olier, G. (2015). Plataforma web “LEGE” para la gestión del consultorio jurídico del Tecnológico Comfenalco. Adelante Ahead, 6(3), 189-198. Recuperado de https://n9.cl/qtlv

INCAP. (s.f.). Sistema de Información. Recuperado de https://n9.cl/xlrf0

Legisoffice. (2014). Legisoffice te permite. Recuperado de https://www.legisoffice.com/\#ax-about

Redelex. (s.f.). Características del software. Recuperado de https://www.redelex.com/caracteristicas/

Siigo. (09 de febrero de 2018). ¿Qué es un sistema de gestión empresarial? Recuperado de https://n9.cl/8g41w Universidad Politécnica de Cataluña. (2004). 7 Sistemas de información. Tecnologías de la información.

Recuperado de https://n9.cl/g4a0j 